Introduction Refinement of intracranial aneurysm rupture risk estimations may improve patient selection for preventive treatment. Dynamical scans may highlight reduced stability of the vessel wall.

Aim of Study The magnitude of pulsations is unknown, but are thought to be smaller than the CT resolution. Noise and artefacts impede reliable measurement. The aim of this study is to quantify the cardiac cycle-related pulsations of intracranial vessels and aneurysms, and to compare patterns of different cardiac cycles.

Methods 4D CTA scans (Aquilion One PRISM Edition, Canon Medical Systems Corporation, Otawara, Japan) of three consecutive heartbeats were obtained in 15 patients harboring an intracranial aneurysm (rotation time $=0.275 \mathrm{~s}$, tube current $=$ $340 \mathrm{~mA}$, tube voltage $=100 \mathrm{kV})$. Reconstructions of every $5 \%$ of the R-R interval were obtained using retrospective cardiac gating. A reference mesh was created per subject to compare segmentations of different cardiac phases. A periodogram was created for the mean pulsatile pattern of a vessel and an aneurysm segment.

Results 15 patients with 19 unruptured and untreated aneurysms with a mean diameter of $5.8 \pm 6.3 \mathrm{~mm}$ (mean \pm std) were scanned, resulting in a mean CTDIvol of 76 [64 94] mGy (mean [min max]). Frequency analysis revealed an artefact with a frequency similar to the gantry rotation frequency. Furthermore, pulsations with frequencies similar to the heart rate were found in the vessel and aneurysm segments.

Conclusions The role of inter-cardiac cycles comparison and frequency analysis is essential for the differentiation between real pulsations and pulsations induced by noise or artefacts on dynamic CTA.

Disclosure Nothing to disclose

\section{EP19 PIPELINE SHIELD FOR THE TREATMENT OF UNRUPTURED INTRACRANIAL ANEURYSMS: LONG-TERM FOLLOW-UP OUTCOMES IN A CASE SERIES OF 67 ANEURYSMS}

J Yeomans, A Sastry. University Hospital of Wales, Cardiff, UK

\subsection{6/neurintsurg-2021-ESMINT.19}

Introduction The Pipeline Flex embolisation device with Shield Technology (Pipeline Shield) is a flow diverter covalently bonded with a polymer proven to reduce thrombogenicity. ${ }^{1}$ Its short- and mid-term safety and efficacy have been established by multiple centres. ${ }^{1}{ }^{2}$ To date, there is no published data regarding its performance beyond 12 months post-procedure.

Objectives To detail the 2-year follow-up outcomes of 67 aneurysms treated with Pipeline Shield in 60 patients.

Aims To discuss the long-term safety and efficacy of Pipeline Shield for unruptured aneurysms.

Methods This prospective, single-arm study assessed 67 aneurysms in 60 patients treated over a 32-month time period from the time of procedure to 2-years post-procedure between October 2017 and May 2020. Data collected included patient demographics, aneurysm features, and intra/periprocedural and 6-month/2-year post-procedural aneurysm appearances and complications.

Results The mean age of the cohort was 58. The majority of aneurysms were small (24/67) or medium (29/67) with a mean aspect ratio of 1.25 and dome to neck ratio of 1.54 .
62 were in the anterior circulation and 5 were posteriorly located. 49 were flow diverted only. The remainder, including all aneurysms larger than $12 \mathrm{~mm}$, were also coiled. All patients were placed on a dual antiplatelet therapy post-procedure regimen. There were no intraprocedural complications and 2 major and 2 minor periprocedural strokes. Satisfactory occlusion was achieved in $87.7 \%(49 / 60)$ of patients at 6 months and 92\% (25/27) at 2 years.

Conclusions Pipeline Shield is safe and efficacious at 2-years follow-up in unruptured aneurysms.

\section{REFERENCES}

1. Trivelato FP, et al. Safety and effectiveness of the pipeline flex embolization device with shield technology for the treatment of intracranial aneurysms: midterm results from a multicenter study. Neurosurgery 2020 Jul 1;87(1):104-111.

2. Yeomans J, et al. Pipeline flex embolisation device with shield technology for the treatment of patients with intracranial aneurysms: periprocedural and 6 month outcomes. Neuroradiol J 2020 Dec;33(6):471-478.

Disclosure Dr A Sastry is a consultant for Medtronic.

\section{EP20 SAFETY AND PERFORMANCE OF THE PENUMBRA SMART COIL SYSTEM FOR PATIENTS WITH POSTERIOR CIRCULATION ANEURYSM}

${ }^{1} \mathrm{C}$ Schirmer, ${ }^{2} \mathrm{~A}$ Spiotta, ${ }^{3} \mathrm{~B}$ Bohnstedt, ${ }^{4} \mathrm{R}$ Bellon, ${ }^{5} \mathrm{H}$ Hawk. ${ }^{7}$ Geisinger Medical Center, Danville, PA; ${ }^{2}$ Medical University of South Carolina, Charleston, SC; ${ }^{3}$ Indiana University Health Physicians (University of Oklahoma Health Sciences Center), Indianapolis, IN; ${ }^{4}$ Swedish Medical Center, Denver, CO; ${ }^{5}$ Erlanger Health System, Chattanooga, TN, USA

\subsection{6/neurintsurg-2021-ESMINT.20}

Introduction Treatment of posterior circulation aneurysm has high rates of morbidity and mortality.

Aim of the Study Evaluate the safety and performance of the SMART COIL System through 1-year follow-up in posterior circulation aneurysm patients enrolled in the SMART registry. Methods This study is a subset analysis of the SMART registry, a prospective, multicenter registry study of 995 patients (905 with aneurysm) treated with SMART COIL, Penumbra COIL 400, or Penumbra Occlusion Device as per the indications.

Results Posterior circulation aneurysm was present in $19.8 \%$ (179/905) of the enrolled patients with aneurysm. Median age of those patients was 62 years, and $78.8 \%$ (141/179) of patients were female. The posterior circulation aneurysm was ruptured in $38.5 \%(69 / 179)$ of patients and wide necked (dome-to-neck ratio $<2$ or neck width $\geq 4 \mathrm{~mm}$ ) in $72.9 \%$ (121/179) of patients. Median packing density of the coiled aneurysms was 28.0\% (IQR 21.4\%-35.2\%).

Raymond Class I or II was achieved in $81.5 \%(145 / 178)$ of posterior circulation aneurysms at immediate post procedure and $88.3 \%(113 / 128)$ at 1-year follow-up. The retreatment rate through 1 year was $6.1 \%(8 / 132)$. The procedural devicerelated serious adverse event rate was $1.7 \%$ (3/179). A modified Rankin Scale score (mRS) of 0 to 2 was observed in $74.7 \%$ (71/95) of patients at 1-year follow-up; from admission to 1-year follow-up, mRS improved or was stable in $75.3 \%$ $(55 / 73)$ of patients.

Conclusions This subset analysis suggests that the SMART COIL System achieves adequate embolization in posterior circulation aneurysms, with a low retreatment rate over 1 year.

Disclosure Clemens Schirmer: Research support: Penumbra; Ownership: NTI. Alejandro Spiotta: Consulting: Stryker, Penumbra, Terumo; Research support: Stryker, Penumbra, 